\title{
An Urban Perinatal Health Programme of Strategies to Improve Perinatal Health
}

\author{
S. Denktaş • G. J. Bonsel • E. J. Van der Weg • A. J. J. Voorham • \\ H. W. Torij · J. P. De Graaf · H. I. J. Wildschut · I. A. Peters · E. Birnie • \\ E. A. P. Steegers
}

Published online: 26 August 2011

(c) The Author(s) 2011. This article is published with open access at Springerlink.com

\begin{abstract}
Promotion of a healthy pregnancy is a top priority of the health care policy in many European countries. Perinatal mortality is an important indicator of the success of this policy. Recently, it was shown that the Netherlands has relatively high perinatal death rates when compared to other European countries. This is in particular true for large cities where perinatal mortality rates are 20-50\% higher than elsewhere. Consequently in the Netherlands, there is heated debate on how to tackle these problems. Without the introduction of measures throughout the entire perinatal health care chain, pregnancy outcomes are difficult to improve. With the support of health care professionals, the City of Rotterdam and the Erasmus University Medical Centre have taken the initiative to develop an urban perinatal health programme called 'Ready for a Baby'. The main objective of this municipal 10 -year programme is to improve perinatal health and to reduce perinatal mortality in all districts to at least the
\end{abstract}

S. Denktaş $(\bowtie) \cdot$ G. J. Bonsel · J. P. De Graaf .

H. I. J. Wildschut - I. A. Peters · E. A. P. Steegers

Division of Obstetrics and Prenatal Medicine, Department

of Obstetrics and Gynaecology, Erasmus University Medical

Centre, P.O. Box 2040, 3000 CA Rotterdam, The Netherlands

e-mail: s.denktas@erasmusmc.nl

E. J. Van der Weg

Municipal Health Service Rotterdam-Rijnmond,

P.O. Box 70032, 3000 LP Rotterdam, The Netherlands

A. J. J. Voorham · H. W. Torij

The School of Midwifery at the Rotterdam University of Applied

Sciences, P.O. Box 2040, 3000 CA Rotterdam, The Netherlands

\section{E. Birnie}

Department of Health Policy \& Management, Erasmus

University, P.O. Box 1732, 3000 DR Rotterdam,

The Netherlands current national average of 10 per 1000 . Key elements are the understanding of the mechanisms of the large health differences between women living in deprived and nondeprived urban areas. Risk guided care, orientation towards shared-care and improvement of collaborations between health care professionals shapes the interventions that are being developed. Major attention is given to the development of methods to improve risk-selection before and during pregnancy and methods to reach low-educated and immigrant groups.

Keywords Urban perinatal health $\cdot$ Structured interventions · Innovation · Pregnancy ·

Perinatal mortality $\cdot$ Health promotion

\section{Background}

Promotion of healthy pregnancies is a top priority of healthcare policy makers in many countries with perinatal mortality being an important index of quality of care. The perinatal mortality rate in the Netherlands is relatively high compared to other European countries [1], which contrasts against the relatively high level of prosperity of this country. The unfavourable position also concerns perinatal morbidity including perinatal conditions related to the probability of perinatal death, such as preterm birth, intrauterine growth restriction, congenital anomalies and a sub-optimal start at birth (e.g. a low Apgar score). There is ample evidence that disrupted intrauterine development negatively affects both short term and long term health of the newborn infant such as postnatal growth and development disorders, psychopathological conditions, diabetes, cardiovascular disease and obesity during childhood and as an adult $[2,3]$. Therefore, perinatal mortality is only the tip 
of the iceberg of adverse perinatal conditions; ill health later in life whether it is during childhood and/or during adult life is the larger part of the iceberg.

Maternal and perinatal health in urban regions considerably differs from the national average, especially the larger cities. In the four largest cities (Rotterdam, Amsterdam, Utrecht and The Hague) perinatal health is poor particular in deprived districts (see Fig. 1). Deprived districts are characterised by a concentration of people with a lower socioeconomic status, single parents and nonwestern immigrants who are poorly integrated in society. Furthermore, their general health is poor when compared to inhabitants of non-deprived neighbourhoods. Relative to the rest of the Netherlands (the Netherlands minus the four large cities) perinatal conditions such as small for gestational age, preterm birth, and perinatal mortality are highest in deprived areas in Rotterdam [4]. Previous research explained these poor perinatal outcomes by the overrepresentation of non-western women, women of low socio-economic status, women living in deprived areas of the city, factors associated with high levels of individual risk factors and with lower performance of care [5].

Two large Dutch cohort studies (i.e., Generation $\mathrm{R}$ in Rotterdam and ABCD in Amsterdam) provided detailed information on the risk factors responsible for poor outcomes and health inequalities between certain groups $[6,7]$. Both the generally increased mortality and morbidity rates and the substantial perinatal health inequalities can be largely attributed to the prevalence distribution of individual risk profiles of pregnant women, and to the suboptimal health performance of perinatal health care services in the Netherlands [8]. The traditional risktailored approach of the Dutch system rests primarily on the principle of an independent risk-assessment and decision making by the midwife, with an emphasis on single reference, rather than shared risk-assessment and responsibility of both the midwife and obstetrician. Secondly, the current system emphasizes medical risks, e.g, prevention is limited to national schemes of screening for STD and blood group antagonism but lifestyle interventions are rare.

Living in deprived neighbourhoods in the larger cities poses additional risks. Here, both non-western and western pregnant women tend to book for antenatal care rather late. For instance, one-third of Moroccan and Antillean women book a visit after 14 weeks of pregnancy, often too late to allow for routine first trimester prenatal screening [9]. The same risk groups hardly make use-up to $80 \%$ - of the post-partum maternity health care services [10].

The role of the potentially modifiable risk factors involved in any reduction of perinatal mortality and morbidity rates depends primarily on successful implementation of effective prevention strategies. A crucial factor is improving recognition that the time immediately before conception and the first trimester of pregnancy are critical for the onset of the majority of foetal abnormalities. The organisation and content of perinatal (primary and secondary care) and public health care should therefore be focused on this early period $[11,12]$.

\section{Reducing Perinatal Mortality in Rotterdam}

In the autumn of 2007 politicians and health care professionals realised from findings from the national obstetric data base that Rotterdam has poor perinatal outcomes. It was also noticed that there was a shortcoming in the collaboration between different health care professionals

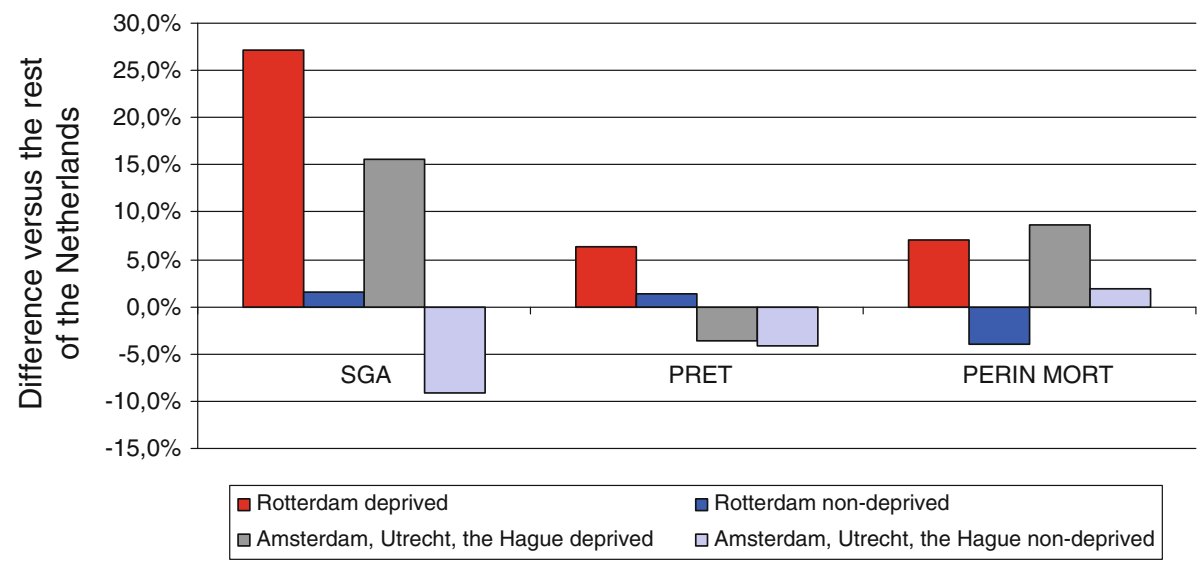

Fig. 1 Differences in perinatal outcome according to deprived or non-deprived districts in Rotterdam and the other three large Dutch cities, as compared to the rest of the Netherlands (adapted from ref nr 5). Data are retrieved from The Netherlands Perinatal Registry and analysed over a 5 year period (2002-2006). SGA Small for
Gestational Age (birth weight below the 10th percentile). PRET preterm birth (gestational age $<37$ weeks). PERIN MORT Perinatal mortality: Foetal death from the 22nd gestational week onwards and early neonatal death (first week post partum) 
resulting in suboptimal quality of health care provided. Together with health scientists of the Erasmus University Medical Centre the Rotterdam municipal council initiated a city wide perinatal health programme to improve perinatal health in Rotterdam.

\section{Outline of the Programme}

The development of the outline of the programme took about 9 months and consisted of several steps. In 2008 it started with an expert meeting with health care professionals, academics and municipal executives to discuss and analyse the poor perinatal health outcomes. The meeting had the format of a focus group in terms of both structure and execution, with each group focusing on a specific issue for an in-depth analysis and discussion. The results of the meeting were as following: (a) determination of areas of special attention, (b) outline of the problem in relation to the desired level of care, (c) delineation of responsibilities of each type of health care professional in the chain of care, (d) analysis for the proposed measures in terms of costs and benefits, (e) identification of innovation options and (f) phasing and overall time schedule for the plan.

A summary of scientific literature about risks, interventions, the differences in socio-economic, ethnic and geographical factors was added to the report of the expert meeting and the draft outline for a monitoring and evaluation plan was made. In a forum that consisted of health care professionals, municipal executives and academics the plan was discussed several times. These forum discussions resulted in a generally approved starting document which provided a framework for the problem analyses, the strategic plan and the envisaged organisational and political form of the municipal programme. In January 2009, this urban perinatal health programme, entitled 'Ready for a baby', was officially launched.

At the core of the organisation is the programme office, consisting of two project managers-one appointed by the municipal health care services and one by the Erasmus University Medical Centre- a number of programme advisers and a communication team. In close collaboration with health care and other professionals working in the field, the programme office formulates and manages selected projects. Professionals involved in a programme project are committed to targets by means of a covenant.

The programme consists of projects that are based on standard care in the obstetrical chain of care and supplemented by a number of non-medical measures. A description of the various components of the programme is elaborated on below.

\section{Link 1: Preconception Care}

Preconception care is world wide recognised as an important part of maternal and child health promotion. It provides the opportunity to prepare future parents for pregnancy in order to optimise their wellbeing and subsequent pregnancy outcomes. The structure of preconception care programs varies between countries depending on their type of health system and level of economic wealth. Programmes and guidelines may also vary in response to local needs. In the past decade interest in preconception care has grown considerably in the Netherlands and the government is looking for ways to implement a structured programme based on preconception care within the regular health services. The Erasmus University Medical Centre has gained much experience with programme-based protocols for preconception care. Experiments with preconception public awareness campaigns have been conducted in the past [e.g. 13]. Still, preconception care remains a relatively new type of care for the public. In 2009, in the North district of Rotterdam a pilot study on preconception care was initiated. Apart from prenatal health benefits the outcome of this pilot study will be a model protocol for preconception care that can be implemented in the whole city. The major challenge of this pilot study is not only to raise public awareness for the concept of preconception care but also to reach the most vulnerable population groups (such as immigrants and those with a low socioeconomic status). One very important aspect is the opportunity to offer preconception care in combination with public health and social welfare services. For this purpose social peer group networks and community social and health workers are involved and channels specific to the target groups are used; these include migrant organisations with peer group educators.

\section{Link 2: Antenatal Care}

World-wide, the general objective of antenatal care is to ensure the best possible pregnancy outcome through optimal preparation and guidance by (a) promoting and maintaining the physical, mental and social health of mother and baby; (b) detecting and managing complications during pregnancy and (c) developing a birth and a complication plan. The structure and organization of antenatal care in the Netherlands differs from most other countries. The Dutch health care system is organised according to the so-called 3-tiered system with clear boundaries between primary, secondary and tertiary levels of care. The midwife is the primary care provider and gatekeeper in the maternity care setting in the Netherlands. Therefore secondary and tertiary levels of care provided by 
obstetricians are only accessible after referral from a midwife. Care for women with an uncomplicated pregnancy will be provided by a midwife without supervision of an obstetrician. If the midwife identifies an increased risk of complications requiring specialist care, she can refer the patient to an obstetrician. In our programme we defined three key issues related to the improvement of the quality of antenatal care. Firstly, there is a disturbing delay among women living in deprived areas in the timing of the booking visit. Secondly, there is relatively high prevalence of various specific medical and social risk factors. Thirdly, a solid motivation for shared care by health care providers is lacking. Therefore, several profound steps need to be taken to improve care.

To start with, public awareness needs to be raised to enhance adequate antenatal care use. Apart from health care professionals social peer group networks and community social workers should be involved. A study into which factors contribute to this delay in antenatal care has just started, a literature search has been done and focus groups are planned in order to develop adequate interventions to tackle this delay in antenatal care. Secondly, preventive and risk-selecting activities during the first trimester of pregnancy need to be intensified. First, a prenatal risk screening instrument-the R4U (Rotterdam Risk Reduction Checklist) - is developed and now tested in a midwifery practice. In addition to the traditional riskselection checklists, the R4U comprises not only medical but also psycho-social and socio-economic risk factors. The addition of these new domains is very relevant because of the risk accumulation resulting in negative pregnancy outcome, especially in disadvantaged urban areas.

Finally, a pilot project of a shared care model including midwives, obstetricians, GP's, social and psychiatric services has been scheduled. By using the R4U, high risk pregnancies can be detected in an early stage of pregnancy. Depending on the risk accumulation, the midwife as case manager at that moment will invite other professionals, sometimes the obstetrician and a psychiatrist and sometimes the GP and social services to discuss the patient and make a shared risk evaluation and treatment plan. The pilot has two aims: (1) to develop a protocol or model for shared care and (2) improve the quality of care and counselling of high risk pregnancies.

\section{Link 3: Birth}

In many industrialized countries, home births declined rapidly over the twentieth century. This decline was in large part due to changes in policies about where birth should take place, an increased migration to large cities and an increased accessibility of hospitals. In the second half of the past century many births in the Netherlands took place at home but declined to less than a third. Home births in Rotterdam declined from $15 \%$ in 2002 to $10 \%$ in 2007 . The resulting shift towards hospital births led to capacity problems in hospitals. Following the initiative of obstetricians, midwives, healthcare insurers and hospital boards, the establishment of primary care birthing centres adjacent to hospitals was recommended. On a new top floor of the Sophia Children's Hospital of the Erasmus University Centre the first birthing centre opened in October of 2010, making it possible for women without a medical indication to give birth in a primary care setting adjacent to the hospital. When specialist care is needed durante partu or right after delivery, it is nearby preventing valuable time loss. In other areas in Rotterdam preparations for similar birthing centres are well under way. These birthing centres provide much more than only a solution for the capacity deficits in the hospitals. In many deprived urban districts, homes are not suitable for a home birth or for the care of mother and baby in the first days following the birth. A birthing centre offers mother and baby a good and safe alternative. After the birth, mother and baby may stay in the birthing centre-with professional maternity care provided $24 \mathrm{~h}$ a day-for at least 4 days. Since the opening of the birthing centre 450 women from various socio-economic and ethnic backgrounds utilised this new type of perinatal health service.

\section{Link 4: Maternity Care}

In many countries care for mother and child in the first days after birth is provided by informal non-professional care givers e.g. family. In the Netherlands maternity care is professionalised as a form of home care services provided by a maternity care assistant. The primary aim is to look after the new mother and her baby during the birth and in the first days afterwards. Complications in the first period after birth can be prevented by proper control and counselling of mother and child. The maternity care assistant may help the parents with her knowledge and experience in areas of baby care, breastfeeding, baby's behaviour etc. In deprived areas of Rotterdam, over $80 \%$ of women do not make use of maternity care services. Probably, many immigrant families are not even aware of - or expect-this kind of home nursing. Even if they are aware of the existence of this facility, they lack the knowledge to arrange it. We have hardly any knowledge about their attitudes and wishes regarding maternity care services which are now mono-cultural oriented. There might very well be a cultural gap between provider of care and the consumer. There are, however, many advantages of using maternity care services and therefore, in our programme, a birthing centre provides 
an excellent opportunity to introduce maternity services to families who are not acquainted with this typical form of Dutch health service. Moreover, maternity services assistants are offered special courses on municipal perinatal health and trained to work in cultural diverse environments. Maternity care providing organisations are involved in policy improvements and interventions to reach target populations. Activities include community focus groups to try to understand the barriers to their use by culturallydiverse women and educating maternity care providers about culturally-diverse patient populations paying attention to culture, traditions and religion.

\section{Link 5: Youth and Family Centres}

Adequate transitions to the Youth and Family Centres run under the auspices of juvenile healthcare in the Netherlands. The Center for Youth and Family is the central point for all questions about parenting and growing up. The center provides advice, guidance and assistance, from the start of pregnancy until the moment a child is grown ( -9 months until 23 years).

Problems that may have arisen during pregnancy, birth or the period after delivery should be communicated to the Youth and Family Centres as soon as and as accurately possible. Often it is possible to identify risk factors that may affect a child's health or upbringing at an early stage (for example, by means of a newborn metabolic screening or a hearing test). Maternity assistants, juvenile healthcare services (youth and family centres, school nurses and doctors), paediatricians, the Youth Care Office or others are able to identify such problems and to take the appropriate steps. Risks that become apparent during a visit to the youth and family centres can also prompt staff to suggest that the mother use preconception counselling before planning a new pregnancy.

\section{Registration and Evaluation}

Registration and evaluation are essential in order to measure whether the objectives of the programme-to improve obstetric outcomes and the healthcare processes-are being achieved. An initial design for the overall evaluation is made; (a) developments will be monitored across the city over a number of years, and (b) project-based evaluations will be carried out. Baseline measurements using registered data have started. Additional information will be collected by means of random surveys. Similar measurements will be collected annually for the next 10 years and reported in a so-called perinatal Atlas of Rotterdam.

\section{Conclusion}

Rotterdam is the first city in the Netherlands, and-to our knowledge - the first in Europe, to introduce measures throughout the entire perinatal health care chain to improve pregnancy outcomes. All professionals involved are convinced that the existing care system must be improved and innovative care processes need to be implemented and evaluated. Evidence-based knowledge on risk factors are of crucial importance in determining new strategies and interventions. The first findings from our own studies suggest that the best results will be achieved from a local approach that is tuned to the local needs, concentrating on the deprived urban areas, in combination with city-wide policies customised to the characteristics of the city itself.

Acknowledgments The urban perinatal health programme 'Ready for a Baby' is conducted by the Erasmus University Medical Centre and the Municipal Health Services Rotterdam Rijnmond, The Netherlands, in close collaboration with the The School of Midwifery at the Rotterdam University of Applied Sciences, the Municipal Centre for Statistics, Youth and Family centres, ACHMEA Health Insurance and the Stichting Trombosedienst \& Artsenlaboratorium Rijnmond (STARMDC). We gratefully acknowledge the contribution of midwives, obstetricians, general practitioners, hospitals, homecare organisations and migrant organisations in Rotterdam, The Netherlands.

Open Access This article is distributed under the terms of the Creative Commons Attribution Noncommercial License which permits any noncommercial use, distribution, and reproduction in any medium, provided the original author(s) and source are credited.

\section{References}

1. Peristat II. (2008). EURO-PERISTAT project in collaboration with SCPE, EUROCAT and EURONEONET. European perinatal health report. Better statistics for better health for pregnant women and their babies in 2004. Available: http://www.europeri stat.com.

2. Barker, D. J. (1995). Fetal origins of coronary heart disease. BMJ, 311, 171-174.

3. Barker, D. J. (2007). The origins of the developmental origins theory. Journal Internal Medicine, 261, 412-417.

4. Poeran, J., Denktas, S., Birnie, E., Bonsel, J., \& Steegers, E. A. P. (2010). Urban perinatal health inequalities. The Journal of Maternal-Fetal and Neonatal Medicine, Early Online, 1-4.

5. Graaf de, J. P., Ravelli, A. C. J., Wildschut, H. I. J., Denktas, S., Voorham, A. J. J., Bonsel, G. J., et al. (2008). Perinatal outcomes in the four largest cities and in deprived neighbourhoods in The Netherlands. Ned Tijdschr Geneeskd, 152(50), 2734-2740.

6. Troe, E. J., Raat, H., Jaddoe, V. W., et al. (2007). Explaining differences in birthweight between ethnic populations. The generation $\mathrm{R}$ study. Bangladesh Journal of Obstetrics \& Gynaecology, 114(12), 1557-1565.

7. Goedhart, G., van Eijsden, M., van der Wal, M. F., \& Bonsel, G. J. (2008). Ethnic differences in preterm birth and its subtypes: the effect of a cumulative risk profile. Bangladesh Journal of Obstetrics \& Gynaecology, 115(6), 710-719.

8. Schutte, J. M., Schuitemaker, N. W., van Roosmalen, J., \& Steegers, E. A. (2008). Dutch maternal mortality committee. 
Substandard care in maternal mortality due to hypertensive disease in pregnancy in the Netherlands. BJOG, 115(6), 732-736.

9. Choté, A. A., Koopmans, G. T., Redekop, W. K., de Groot, C. J., Hoefman, R. J., Jaddoe, V. W., et al. (2011). Explaining ethnic differences in late antenatal care entry by predisposing, enabling and need factors in the Netherlands. The generation R study. Maternal and Child Health Journal, 15(6), 689-699.

10. Steegers, E. A. P., De Graaf, J. P., Laudy, J. A. M., Voorham, T., Happel, M., Denktaş, S., et al. (2008). Right to a healthy start [in Dutch: Recht op een goede start]. Med Contact, 63:100-101.

11. Atrash, H. K., Johnson, K., Adams, M., et al. (2006). Preconception care for improving outcomes: the time to act. Maternal and Child Health Journal, 10, S3-S11.
12. Korenbrot, C. C., Steinberg, A., Bender, C., \& Newberry, S. (2002). Preconception care: A systematic review. Maternal and Child Health Journal, 6(2), 75-88.

13. Laudy, J. A. M., Elsinga, J., Wildschut, H. I. J., \& Steegers, E. A. P. (2007). Opzet en organisatie van preconceptiezorg: een Rotterdams model voor preconceptiezorg. Eerst een verhaaltje en dan naar bed. In E. Slager (Ed.), Reproductieve geneeskunde, gynaecologie en obstetrie anno 2007 (pp. 181-186). Haarlem: DCHG Medische Uitg. 